\title{
Simulation du coup de vent latéral Attentes du secteur automobile et état de l'art
}

\author{
Simulation of the lateral gust of wind \\ Automotive expectations and state of the art
}

par P. Gilliéron

Direction de la Recherche, Renault

F. Chometon

Conservatoire National des Arts et Métiers, Paris

The means of prediction of the unsteady aerodynamic effects associated with gust of wind are rare and difficult to develop. This paper proposes a state of the art on the experimental and numerical techniques intended to improve comprehension of the physical phenomena and to quantify the effects of the gust of the wind. For each solution, the experimental andlor modelisation conditions are recalled and the principal results presented.

\section{INTRODUCTION}

La stabilité dynamique des véhicules automobiles est fortement influencée par l'évolution de leurs caractéristiques aérodynamiques lors des phases de croisement, de dépassement, d'entrée ou de sortie de tunnel en présence de vent latéral constant ou variable en intensité, sens et direction. La distribution des pressions à la surface du véhicule change rapidement et soumet le véhicule à de rapides sollicitations autour des axes de lacet, de roulis et de tangage. La trajectoire du véhicule évolue et le conducteur effectue de brèves corrections de trajectoire.

Ces phénomènes transitoires, généralement bien perçus par les automobilistes, sont des sources d'inconfort et d'insécurité. En l'absence de vent latéral et lors du dépassement d'un convoi avec remorque par une berline, les pressions statiques pariétales peuvent atteindre des niveaux proches des valeurs relevées en stationnaire pour des angles de dérapage voisins de 10 degrés. En sortie de tunnel et en présence de vent latéral constant, la valeur du moment de lacet d'un véhicule de type berline peut dépasser de $30 \%$ les valeurs relevées en stationnaire, Bourdassol [1]. Concernant le caractère instationnaire du vent au voisinage du sol, des relevés anémométriques montrent que le spectre des rafales de vent présente un maximum compris entre 0,3 et 1,0 hertz [2]. Le conducteur est alors soumis à de rapides modifications de son environnement visuel autour de l'axe de lacet et à de fortes variations du bruit acoustique.

Après avoir présenté les attentes du secteur automobile, cet article propose de rappeler l'effet du vent latéral sur la trajectoire du véhicule, puis passe en revue les principales techniques de mesure et d'analyse associées à sa modélisation stationnaire et instationnaire. Pour chaque approche, les résultats principaux sont commentés.

\section{II - ATTENTES DU SECTEUR AUTOMO- BILE}

Dans le domaine automobile, les moyens de prévision des sollicitations aérodynamiques instationnaires sont rares et souvent difficiles à mettre en œuvre. Les ingénieurs sont donc relativement démunis pour évaluer le comportement dynamique d'un véhicule lors des phases de croisement, de dépassement, d'entrée et de sortie de tunnel en présence de vent latéral ou encore lorsque le véhicule est soumis à un coup de vent latéral.

Les attentes des secteurs opérationnels de l'automobile portent essentiellement sur la compréhension des mécanismes physiques associés aux phénomènes instationnaires en vue d'améliorer la stabilité dynamique des véhicules. Il s'agit également de déterminer les efforts instationnaires qui s'exercent sur certains éléments de carrosserie (vitres laté- 
rales, attaches de rétroviseur, ...) lors des phases de croisement, de dépassement, d'entrée ou de sortie de tunnel. Ces attentes nécessitent le développement de nouvelles techniques de mesure, de nouvelles techniques de visualisations instationnaires et de nouvelles approches numériques.

\section{III — BASES THÉORIQUES}

Lorsqu'un véhicule animé d'une vitesse $\vec{V}_{0}$ subit un vent latéral $\vec{W}$, il est soumis à un vent apparent $\vec{V}_{r}$ auquel correspond un angle de dérapage $\beta$ (fig. 1). Le torseur aérodynamique résultant $\left(F_{x}, F_{y}, F_{z}, L, M, N\right)$ participe à la déformation des pneumatiques. Les efforts $F_{x}, F_{y}$ et $F_{z}$ désignent respectivement la traînée, l'effort latéral et la portance aérodynamiques. Les moments $L, M$ et $N$ sont les moments de roulis, de tangage et de lacet. A chaque pneumatique est conventionnellement associée une vitesse de dérive $\vec{V}_{t}$ et un angle de dérive $\delta$, (fig. 1 ). La composante $F_{y p}$ de la force de contact du pneu et du sol est appelée poussée de dérive du pneumatique. Pour une résultante verticale donnée et un coefficient de frottement $C_{f}$ l'évolution de l'angle de dérive

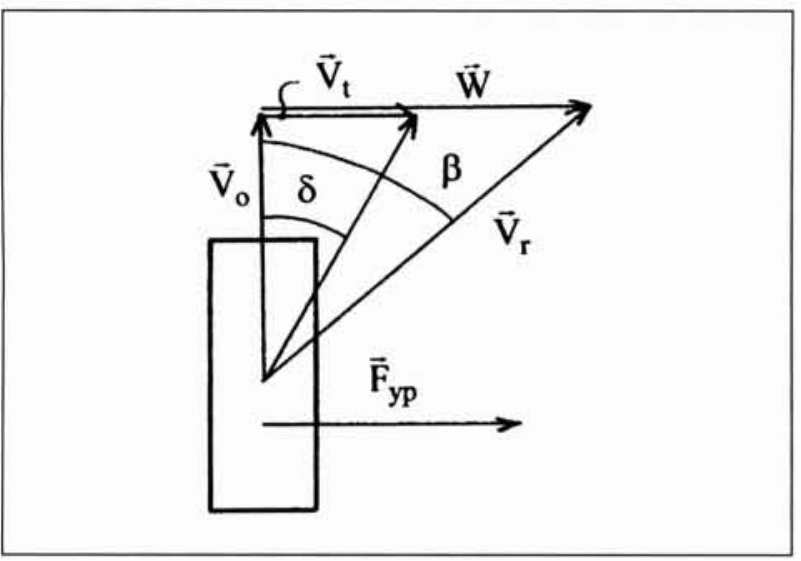

1. $\delta$, angle de dérive du pneumatique, $\beta$, angle de dérapage du véhicule.

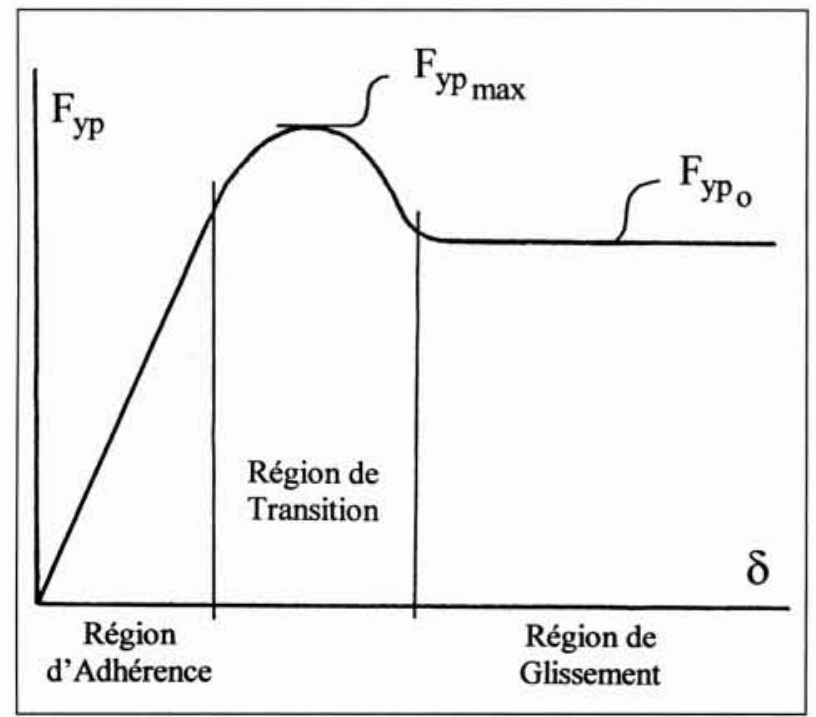

2. Evolution de l'angle de dérive $\delta$ en fonction de la poussée de dérive $F_{y p}$. $\delta$ en fonction de la poussée de dérive $F_{y p}$ est représentée figure 2. L'analyse des résultats permet de définir une région d'adhérence et une région de glissement situées de part et d'autre d'une région de transition. Dans la région d'adhérence du pneumatique, l'angle de dérive est faible et varie linéairement en fonction de la poussée de dérive. Au-delà de $F_{y p \max }$ et tant que l'effort latéral reste supérieur à $F_{y p 0}$, l'angle de dérive augmente. Par la suite, la vitesse de dérive $\vec{V}_{t}$ est négligée par rapport au vent latéral $\vec{W}$.

\section{IV — APPROCHE EXPÉRIMENTALE}

Dans les développements qui suivent les coefficients adimensionnels $C_{x}, C_{y}, C_{z}, C_{L}, C_{M}$ et $C_{N}$ associés au torseur aérodynamique $\left(F_{x}, F_{y}, F_{z}, L, M, N\right)$ désignent respectivement les coefficients aéródynamiques de traînée, d'effort latéral, de portance, de roulis, de tangage et de lacet appliqués au véhicule. A titre d'exemple, le coefficient de traînée aérodynamique est défini par :

$$
C_{x}=\frac{F_{x}}{\frac{\rho}{2} V_{0}^{2} S} .
$$

Dans cette expression, $\rho$ est la masse volumique du fluide et $S$ le maître couple du véhicule.

D'un point de vue expérimental, l'approche la plus simple consiste à déterminer en soufflerie les caractéristiques aérodynamiques statiques sur des véhicules à échelle grandeur ou sur maquettes. Les expérimentations s'effectuent pour différentes valeurs de l'angle de dérapage $\beta$. A titre d'exemple, les produits du maître couple $S$ par les coefficients aérodynamiques d'efforts et de moments relevés sur un véhicule échelle 1 de type berline en faisant varier l'angle de dérapage de 0 à 14 degrés sont reportés sur les figures 3 et 4 . Ces coefficients aérodynamiques statiques peuvent être utilisés dans les modèles numériques de stabilité dynamique. Ce type d'approche ne prend cependant pas en compte le caractère instationnaire de l'écoulement associé au coup de vent latéral et des essais dynamiques sont nécessaires. Ces essais s'effectuent sur des véhicules à échelle grandeur ou sur maquettes.

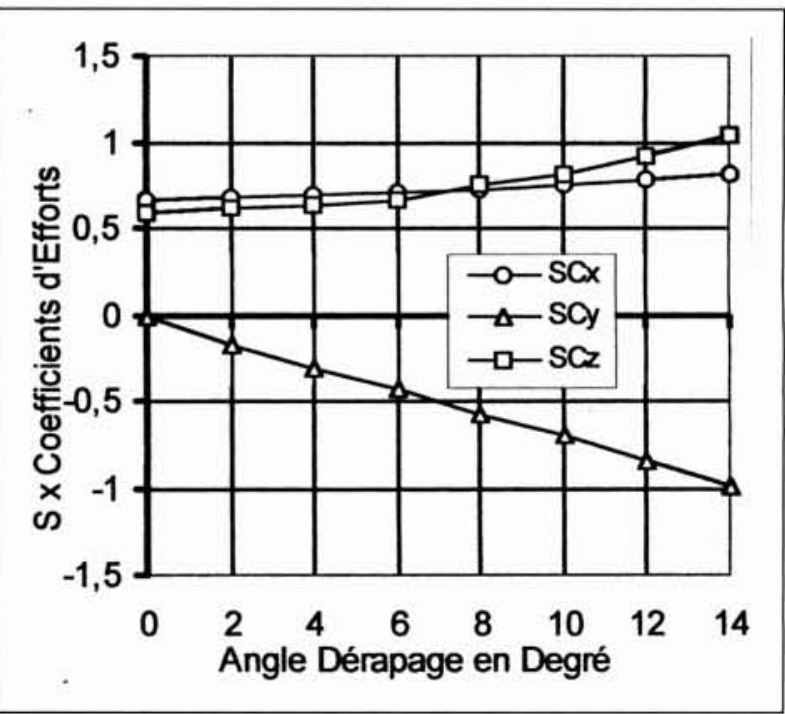

3. Produits des Coefficients d'Efforts par le maître couple $S$ du véhicule. 


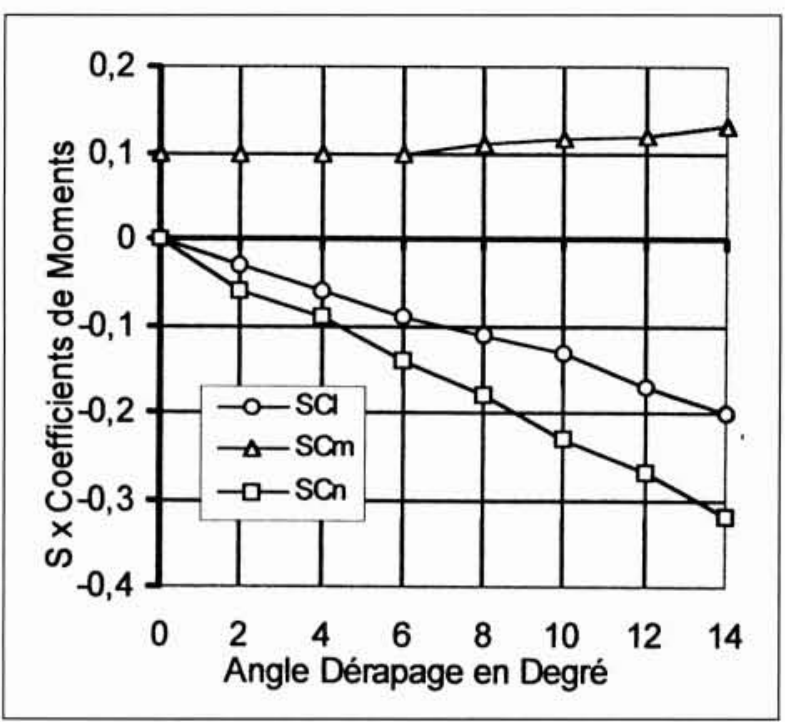

4. Produits des Coefficients de Moments par le maître couple $S$ du véhicule.

\subsection{Essais dynamiques à échelle grandeur}

Une solution consiste à déplacer le véhicule en ligne droite à vitesse stabilisée [3]. Des ventilateurs fixes, positionnés dans des plans transversaux à la direction du véhicule, simulent une rafale de vent latéral. Ces expérimentations permettent de mettre en évidence les limites de renversement des remorques ou encore de quantifier des écarts et/ou des corrections de trajectoire. Les résultats sont fortement influencés par les réactions du conducteur et les caractéristiques mécaniques des véhicules. Compte tenu des coûts d'appareillage, ce type d'approche ne permet généralement pas d'estimer l'évolution des coefficients aérodynamiques et des distributions des pressions statiques pariétales. Une analyse du comportement dynamique des véhicules est donnée par MacAdam et al. [4].

La détermination des coefficients aérodynamiques pour deux ou plusieurs véhicules se déplaçant à faible distance relève également de cette problématique. Les travaux de Hong et al. [5] portent sur l'analyse expérimentale de l'évolution de la traînée aérodynamique de deux monocorps se déplaçant en tandem. Les résultats présentés portent sur l'évolution de la trainée aérodynamique des véhicules de tête et de queue en faisant varier leur distance de séparation. Les résultats à échelle grandeur sont comparés à des résultats obtenus en soufflerie sur des maquettes à échelle $1 / 8$. Pour les véhicules à échelle grandeur, les traînées aérodynamiques sont mesurées à partir des forces d'inertie. Les phases d'accélération et de décélération permettent d'accéder respectivement à la traînée aérodynamique du véhicule de queue et de tête. Le nombre de Reynolds de l'écoulement basé sur la longueur des véhicules est proche de $10^{6}$. Les résultats obtenus sur maquettes à échelle réduite sont en bon accord avec les résultats obtenus sur route. En particulier, l'évolution du phénomène physique est bien reproduite en soufflerie. Les trainées relevées sur véhicules à échelle grandeur sont supérieures aux valeurs relevées en soufflerie avec des erreurs proches de $10 \%$.

\section{- 4.2 Essais dynamiques sur maquettes}

L'effet d'un coup de vent latéral constitue la configuration la plus contraignante du point de vue aérodynamique. Cette situation est caractéristique d'une sortie de tunnel en pré-

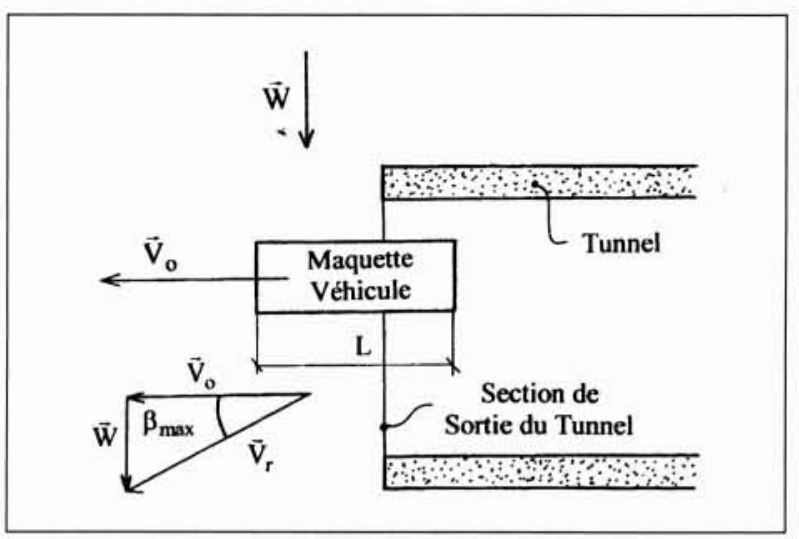

5. Véhicule en sortie de tunnel.

sence de vent latéral ou encore du croisement ou du dépassement de deux véhicules avec ou sans vent latéral.

En désignant respectivement par $V_{0}$ et $L$ la vitesse et la longueur du véhicule, l'intervalle de temps associé à la traversée de la section de sortie d'un tunnel est donné par (fig. 5):

$$
\Delta t=\frac{L}{V_{0}}
$$

Si $\beta_{\text {max }}$ est l'angle de dérapage défini pour un essai statique, alors durant l'intervalle de temps $\Delta t$, l'angle de dérapage $\beta$ évolue de 0 à $\beta_{\max }$. Durant ce temps $\Delta t$, l'information $\beta$ est transportée à la vitesse $V_{0}$ sur la longueur $L$ du véhicule. Expérimentalement ce type de phénomène peut être analysé comme la réponse du véhicule à une sollicitation du type échelon (fig. 6). A cette sollicitation peut être associée un mouvement harmonique de période $T=4 \Delta t$ et de fréquence :

$$
f=\frac{V_{0}}{4 L}
$$

Pour un véhicule automobile de longueur $L=4$ mètres se déplaçant à la vitesse $V_{0}=90 \mathrm{~km} / \mathrm{h}$, la fréquence du mouvement harmonique est proche de 1,6 hertz. En sortie de phase transitoire et pour un vent traversier $W$ égal à $20 \mathrm{~km} / \mathrm{h}$, l'angle de dérapage $\beta_{\max }$ est égal à 12,5 degrés. Lors d'un croisement, la vitesse $V_{0}$ s'identifie à la somme des vitesses

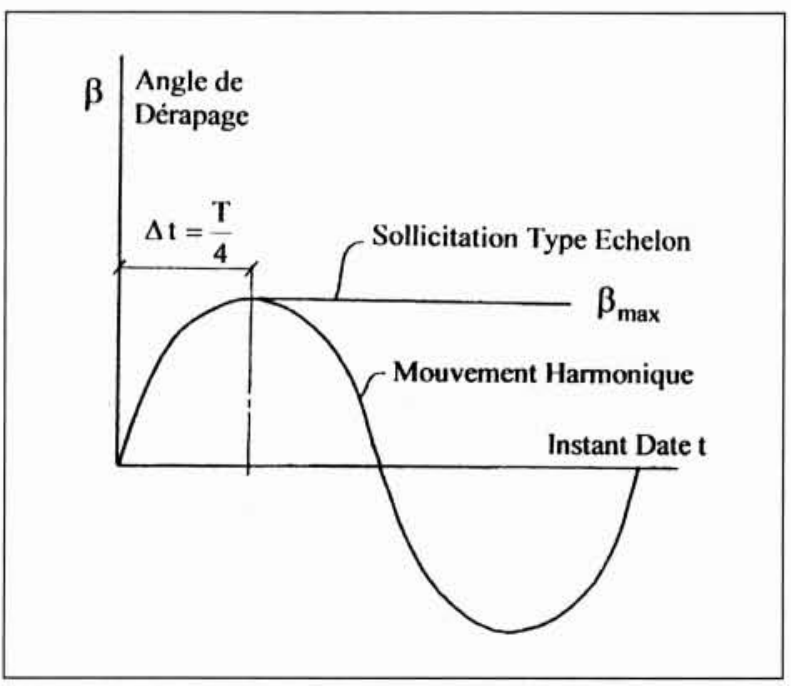

6. Angle de dérapage $\beta$ en fonction du temps. 
des deux véhicules. Pour deux véhicules de longueur $L=4$ mètres se déplaçant à la vitesse de $90 \mathrm{~km} / \mathrm{h}$, la fréquence du mouvement harmonique définie par (2) est de l'ordre de 3 hertz. Pour un dépassement, le calcul est effectué avec la différence des vitesses. Lorsque l'expérimentation doit s'effectuer en soufflerie sur une maquette à échelle réduite, la vitesse $V_{0}$ augmente pour satisfaire les contraintes associées au nombre de Reynolds. A titre d'exemple, pour une vitesse $V_{0}$ égale à $140 \mathrm{~km} / \mathrm{h}$ et une maquette de longueur $L=1$ mètre, cette fréquence est proche de 10 hertz. Ce niveau de fréquence, associé à la même amplitude $\beta_{\max }$, rend l'expérimentation mécaniquement difficile.

Différentes approches sont alors proposées. Une première solution consiste à catapulter le véhicule perpendiculairement à la direction de l'écoulement incident. Des expérimentations effectuées par Macklin et al. de l'Université de Cranfield USA [6] sur des maquettes de véhicules automobiles permettent d'analyser les écarts entre les valeurs statiques et dynamiques des efforts latéraux et de portance d'une part et des moments de lacet et de roulis d'autre part. La catapulte est constituée d'un système élastique et la vitesse de déplacement de la maquette est identique pour toutes les configurations. Pour les essais dynamiques, les différents angles de dérapage s'obtiennent en faisant varier la vitesse de l'écoulement incident $W$. Pour des angles de dérapage $\beta$ égaux à 0 et 45 degrés, le nombre de Reynolds varie de $3,4 \cdot 10^{5}$ à $4,7 \cdot 10^{5}$. Pour des angles de dérapage inférieurs à 15 degrés, les résultats statiques et dynamiques sont très similaires. Au-delà de 15 degrés, les valeurs dynamiques des efforts et des moments deviennent plus importantes que les valeurs obtenues lors des essais statiques.

Une seconde solution consiste à soumettre la maquette fixe à un flux généré par des profils oscillants disposés en amont [7]. Le dispositif permet de modéliser un écoulement périodique d'amplitude 15 degrés. La fréquence d'oscillation maximale est de 25 hertz et le nombre de Reynolds est égal à $4,5 \cdot 10^{5}$. Les maquettes expérimentées sont du type corps de Davis [8]. La variation de l'angle de dérapage s'obtient en faisant varier la vitesse de l'écoulement infini amont. Les résultats instationnaires obtenus par les auteurs, comparés à des résultats stationnaires, montrent que les efforts peuvent être évalués simplement en supposant que l'écoulement se comporte comme un écoulement quasi-stationnaire.

Une autre solution proposée par Garry et Cooper [9] consiste à utiliser une maquette mise en rotation autour de son axe vertical. Les expérimentations s'effectuent sur une géométrie simplifiée à culot droit. Une balance permet d'effectuer des relevés stationnaires et instationnaires. Les résultats portent sur l'analyse et la comparaison des efforts latéraux, des traînées et des moments de lacet. La fréquence d'oscillation est inférieure ou égale à 0,4 hertz et l'amplitude du mouvement égale à 40 degrés. Le nombre de Reynolds est de 0,5 . $10^{6}$. Les amplitudes de la traînée et du moment de lacet stationnaire et instationnaire restent du même ordre de grandeur. Par contre, les valeurs des coefficients de trainée stationnaires et instationnaires présentent un déphasage fonction de la géométrie. Ce déphasage apparaît même à faible fréquence.

Ces résultats sont complétés par une analyse des structures tourbillonnaires de sillage [10]. L'analyse s'effectue par post-traitement des pertes de pression d'arrêt relevées dans les sillages en aval du culot dans des plans transversaux perpendiculaires à la direction de l'écoulement amont. Les pertes de pression d'arrêt sont relevées à l'aide d'une sonde de Kiel dynamique. La fréquence d'oscillation de la maquette est de 1 hertz, l'amplitude du mouvement est égale à 10 degrés et le nombre de Reynolds rapporté à la longueur du véhicule est de $1,6 \cdot 10^{6}$. Les méthodes d'acquisition et de post-traitement basées sur l'utilisation des polynômes de Tchebychev permettent de visualiser l'évolution du champ de pression instationnaire et de reconstituer un film. Les résultats permettent de mettre en évidence et d'améliorer la compréhension des phénomènes liés au déphasage entre les différentes structures tourbillonnaires. Aucun phénomène d'hystérésis n'est mis en évidence.

Ces résultats sont obtenus dans des souffleries à veine ouverte et semi-guidée. Pour les fortes valeurs de l'angle de dérapage $\beta$, l'écoulement autour du véhicule et dans le sillage peut subir l'influence de l'écoulement de retour. La distribution de pression autour du véhicule n'est alors pas représentative des conditions réelles. Pour résoudre ce problème, Dominy et al. [11] proposent une nouvelle approche. La maquette, disposée dans une veine ouverte sur l'extérieur, est soumise à un jet incident dirigé suivant l'axe longitudinal du véhicule. La soufflerie est sans retour. Un second jet, disposé latéralement, permet de générer un écoulement traversier $W$. Un dispositif commande l'ouverture et la fermeture de l'orifice de sortie du flux d'air traversier sur une durée variable. Ce dispositif, où la maquette est fixe, permet de s'affranchir des problèmes d'inertie, de mesurer le torseur aérodynamique et d'effectuer en parallèle des relevés de vitesse et de pression instationnaires dans les sillages. Les phénomènes physiques attachés au coup de vent latéral sont alors mieux reproduits. Le nombre de Reynolds de l'écoulement, la fréquence du coup de vent latéral et l'angle de dérapage peuvent atteindre respectivement $2.10^{5}, 10$ hertz et 35 degrés.

Les effets associés au dépassement de véhicules sont étudiés par Minato et al. [12]. L'analyse s'effectue à partir de mesures d'efforts, de pressions et de visualisations à l'aide de plans lasers associés à un post-traitement d'images. Les effets les plus significatifs apparaissent lorsque le véhicule dépassant arrive à proximité de l'arrière du véhicule dépassé. Dans cette situation, la traînée aérodynamique du véhicule dépassé augmente et les structures de sillage évoluent rapidement. Les résultats sont comparés à ceux obtenus par une méthode numérique de type volumes finis associée à un modèle de turbulence $k-\varepsilon$.

Afin de compléter ces résultats, l'Institut Aérotechnique de Saint-Cyr-l'Ecole et le Laboratoire d'Aérodynamique du Conservatoire National des Arts et Métiers sont actuellement associés sur un programme de recherche visant à améliorer la compréhension des phénomènes physiques liés au dépassement. L'objectif est de mesurer le torseur aérodynamique instationnaire appliqué à un véhicule soumis à l'influence d'un véhicule dépassant. Les essais s'effectuent sur des maquettes à échelles $1 / 5$ et le nombre de Reynolds est de $3,6 \cdot 10^{6}$. Le dépassement s'obtient à partir d'une maquette fixe et d'une maquette animée d'un mouvement uniforme de translation. La maquette fixe est reliée à une balance aérodynamique. Le dispositif permet également de simuler l'effet d'un vent latéral sur les deux véhicules.

Dans le même esprit, des expérimentations récentes ont été effectuées sur un train de trois maquettes identiques [13]. Les résultats sont obtenus sur maquettes à échelle $1 / 8$ pour un angle de dérapage égal à 10 degrés. Le nombre de Reynolds est égal à $4.10^{5}$. L'analyse porte sur la comparaison des coefficients de traînée, des efforts latéraux et des moments de lacet en faisant varier la distance entre chaque maquette. La traînée du véhicule de queue est supérieure à la traînée du véhicule de tête et la position intermédiaire apparaît la plus favorable. Pour les faibles distances de séparation, la traînée du véhicule de tête est proche de la traînée du véhicule situé en position intermédiaire. Lorsque cette distance augmente, le phénomène s'inverse et la traînée aérodynamique de la maquette de tête devient proche de la traînée du véhicule de queue. Les efforts latéraux et les moments de lacet les plus importants et les plus faibles sont respectivement relevés sur les maquettes de tête et de queue.

\section{APPROCHES NUMÉRIQUES}

Dans un contexte industriel, la modélisation des écoulements aérodynamiques s'effectue à l'aide de méthodes de type 
RANS (Reynolds Average Navier Stokes) associées à des modèles de turbulence avec ou sans loi de paroi. Le modèle le plus utilisé pour modéliser les écoulements en aérodynamique externe est le modèle de type $k-\varepsilon$ avec loi de paroi logarithmique. Le nombre de cellules volumiques associé au domaine fluide est aujourd'hui généralement compris entre 2 et 10 millions d'éléments, ce dernier chiffre ne correspondant généralement pas à une configuration de type industrielle. Les maillages peuvent être structurés ou non structurés mais les maillages structurés restent aujourd'hui rarement utilisés compte tenu de leurs durées de réalisation.

En écoulement stationnaire, le stade du calcul prédictif des coefficients aérodynamiques pour un véhicule réel n'est pas atteint. Des résultats intéressants peuvent cependant être obtenus sur des géométries simplifiées à formes anguleuses. Des comparaisons calculs - mesures sont effectuées par Taeyoung et al. [14] (General Motors, 1996) sur des géométries simplifiées proches de la géométrie du corps de Ahmed, [15]. Le nombre de Reynolds expérimental rapporté à la longueur des maquettes est égal à $1,24 \cdot 10^{6}$. La comparaison porte sur la traînée aérodynamique, la portance et sur la répartition des coefficients de pression pariétale. La turbulence est modélisée à l'aide des modèles $k-\varepsilon$ et RNG $k-\varepsilon$ (ReNormalization Group). Les valeurs des coefficients aérodynamiques issus du calcul sont supérieures aux valeurs expérimentales et l'écart diminue avec la densité du maillage. Pour le modèle $k-\varepsilon$, les différences entre les valeurs expérimentales et numériques sont au mieux de l'ordre de $13 \%$. Pour le modèle RNG $k-\varepsilon$, ces différences sont proches de $4 \%$. L'influence du nombre de Reynolds est également analysée. Les phénomènes physiques associés aux différences géométriques des maquettes semblent bien reproduits par le calcul. Le travail ne fournit aucune information sur les lignes de frottement et les structures tourbillonnaires de sillage.

Plus récemment, des expérimentations numériques ont été effectuées sur le corps de Ahmed en faisant varier l'inclinaison de la lunette arrière [16]. Réalisé à partir de contraintes industrielles, le maillage volumique constitué de prismes et de tétraèdres ne comprend que $5.10^{5}$ éléments. Le nombre de Reynolds de l'écoulement est égal à $4 \cdot 10^{6}$. Les résultats montrent qu'il est possible de reproduire la topologie des lignes de frottement et la position des décollements. Les structures tourbillonnaires et l'évolution du coefficient de traînée aérodynamique lors des phases de transition entre les structures bidimensionnelles de type culot droit et les structures tridimensionnelles de type bicorps sont bien représentées. Ces résultats s'obtiennent en respectant des règles physiques sur la structure du maillage au voisinage de la paroi et sur les gradients de pression dans les sillages. En particulier, les valeurs des y+ sont voisines de 30 . Les différences relevées entre les coefficients de traînée aérodynamique numériques et expérimentaux en faisant varier l'inclinaison de la lunette arrière de 0 (culot droit) à 30 degrés varient de $35 \%$ à $0 \%$.

En général la comparaison des résultats de calculs et expérimentaux fait apparaitre des différences au niveau du développement des structures tourbillonnaires émises dans les sillages, des distributions de pression statique au culot et par conséquent sur la traînée aérodynamique. L'origine de ces écarts est principalement liée à une mauvaise restitution de l'écoulement dans le volume de proximité de paroi. Les lois de paroi et les modèles de turbulence sont en effet utilisés sur des configurations géométriques pour lesquelles ils ne s'appliquent que très difficilement. Des préconisations de maillage, de schémas numériques et de modèles de turbulence $k-\varepsilon$, RNG $k-\varepsilon$ et RSM sont fournies par Perzon et al. [17] (Volvo). L'augmentation du nombre de mailles permet de réduire ces écarts mais la durée de réalisation des maillages peut devenir incompatible avec les exigences industrielles.

Lorsque les formes des véhicules deviennent arrondies, la géométrie ne permet plus de piloter la position des décolle- ments, Gilliéron 1999 [18]. Numériquement, la position des lignes de décollement se situe en aval de la position réelle. Certaines d'entre elles peuvent ne plus être reproduites par le calcul. A ces difficultés de représentativité s'ajoutent des difficultés de génération des maillages volumiques. A titre d'exemple et lorsque les modèles doivent intégrer des éléments géométriques de faibles dimensions comme les rétroviseurs, les contraintes imposées au maillage à proximité de la paroi deviennent trop importantes et la génération du maillage volumique devient impossible dans un contexte industriel.

Concernant la simulation du vent latéral, les calculs en statique sont actuellement les plus faciles à effectuer. A titre d'exemple, les travaux effectués par Okumura et al. [19] sur des géométries de type culot droit en faisant varier l'angle de dérapage de 0 à 45 degrés permettent de comparer les évolutions des efforts latéraux calculés et expérimentaux. Le maillage utilisé est symétrique et correspond au maillage utilisé pour le calcul sans dérapage. Le nombre de Reynolds de l'écoulement est égal à $2.10^{6}$ et le vent latéral est simulé à partir de conditions limites imposées à l'entrée du domaine fluide. Le modèle numérique comprend $1,1.10^{5}$ éléments de type hexaèdres et l'écoulement turbulent est modélisé à l'aide d'un modèle $k-\varepsilon$ avec loi de paroi logarithmique. Les valeurs de $y+$ sont proches de 40 . L'étude a permis d'optimiser la géométrie du bouclier avant par réduction des structures tourbillonnaires émises sous le vent. L'optimisation d'un pré et d'un post-processeur permet de faire chuter les durées de développement de 3 semaines à 4 jours (Daihatsu Motor Co).

Des expérimentations de même nature sont aujourd'hui en cours de réalisation au sein de la société Renault. Elles constituent une première étape vers la caractérisation des phénomènes instationnaires liés au coup de vent latéral. Les simulations numériques s'effectuent sur le corps de Ahmed pour différents angles de dérapage. L'angle compris entre la lunette arrière et l'horizontale est égal à 30 degrés. Cette géométrie permet de fixer la position des décollements sur l'arrière du véhicule et de s'affranchir localement des limites structurelles des modèles de turbulence associés aux approches de type RANS. Le maillage volumique réalisé à partir de contraintes industrielles est constitué de prismes et de tétraèdres [16]. Il peut comprendre jusqu'à $2 \cdot 10^{6}$ éléments et le nombre de Reynolds de l'écoulement est de $2,6 \cdot 10^{6}$. L'objectif est ici de caractériser les effets du vent latéral sur l'évolution des lignes de frottement, des structures tourbillonnaires de sillage, sur la traînée aérodynamique et sur l'effort latéral. Les structures tourbillonnaires de sillage sont analysées à l'aide de cartographies de vitesses et de pertes de pression d'arrêt. Les résultats numériques comparés aux résultats expérimentaux doivent permettre de mettre au point une approche calcul et d'en identifier les limites.

Ces travaux sont complétés par des calculs destinés à caractériser les effets instationnaires associés à un mouvement harmonique. La fréquence du mouvement est égale à 1 hertz et l'amplitude angulaire $\beta_{\max }$ égale à 10 degrés. Le nombre de Reynolds de l'écoulement vaut $1,6.10^{6}$. La méthodologie s'appuie sur l'utilisation d'un code industriel de type RANS et s'inscrit en prolongement des travaux effectués par Gilliéron et al. [16] et Chometon et al. [10]. Le modèle de turbulence utilisé est du type $k-\varepsilon$ avec loi de paroi logarithmique. Le véhicule est contenu à l'intérieur d'un cylindre d'axe vertical. Le domaine fluide et le véhicule, intérieurs au cylindre, sont animés d'un mouvement harmonique de rotation. Des expérimentations en soufflerie, prévues dans le cadre d'un projet PREDIT, doivent permettre d'effectuer des comparaisons avec les résultats issus du calcul.

\section{VI $\square$ CONCLUSION}

Les attentes du secteur automobile en termes de compréhension des phénomènes physiques et d'analyse des effets du 
vent latéral sont présentées. Les concepts théoriques et les principaux résultats expérimentaux et numériques sont ensuite rappelés. En général, les essais à échelle grandeur restent difficiles et coûteux à mettre en œuvre. Les expérimentateurs privilégient alors les essais sur maquettes à échelle réduite. La maquette peut être catapultée, soumise à un mouvement harmonique autour de son axe de lacet, fixe et soumise à un vent oscillant ou encore fixe et soumise à un vent latéral de type échelon. Pour chaque technique, l'analyse des phénomènes physiques s'effectue à partir de la mesure du torseur aérodynamique appliquée à la maquette et/ou des pressions ou des vitesses relevées dans les sillages. En permettant de s'affranchir des problèmes d'inertie liés aux balances embarquées dans les maquettes, la solution proposée par Dominy et al. [12] apparaît cependant la plus intéressante.

S'agissant des approches numériques, les modèles de turbulence implantés dans les logiciels industriels restent aujourd'hui insuffisants pour modéliser de manière satisfaisante l'ensemble des écoulements tridimensionnels décollés qui prennent naissance autour des véhicules automobiles. Seule la simulation autour de formes géométriques simplifiées à arêtes vives peut être envisagée. Les expérimentations stationnaires simulant l'effet d'un vent latéral uniforme constant en direction, sens et module restent les plus faciles à obtenir.

\section{REMERCIEMENTS}

Ce travail a été partiellement réalisé dans le cadre de la convention de recherche à partenaires multiples $n^{\circ} 98 \mathrm{~A}$ 0249 du programme national PREDIT2 intitulée "Aérodynamique Instationnaire des Véhicules Terrestres et de leur Environnement". Les partenaires de cette convention sont la SNCF, RENAULT, PRINCIPIA et l'Ecole Nationale Supérieure de Mécanique et d'Aérotechnique de Poitiers.

\section{RÉFÉRENCES}

[1] BouRdassol C. (1996). - Analyse de l'excitation aérodynamique en lacet d'un véhicule soumis à une rafale de vent, Journées d'Etudes «Aérodynamique-Aéroacoustique-Aérothermique Automobile et Ferroviaire ", Société des Ingénieurs de l'Automobile, 5 et 6 novembre, Courbevoie Paris.

[2] Traité de Physique du Bâtiment, Tome 1, Connaissances de Base, Centre Scientifique et Technique du Bâtiment, ISBN 2-86891-240-0.

[3] Ниснот W.H. (1998). - Aerodynamics of Road vehicles, Fourth Edition, SAE International, pp. 300-306, ISBN 07680-0029-7

[4] Macdam C.C., Sayers M.W., Pointer J.D. and Gleason M. - Crosswind Sensitivity of Passenger Cars and the Influence of Chassis and Aerodynamic Properties on Driver Preferences, Vehicle Aerodynamics, SAE International, PT49, pp. 281-315, ISBN 1-56091-594-3.

[5] Hong P., Marcu B., Browand F. and Tucker A. (1998). - Drag Forces Experienced by Two, Full-Scale Vehicles at Close Spacing, SAE paper 980396, International Congress, Detroit, USA.
[6] MaCkLin A.R., GarRY K.P. and Howell J.P. (1996). Comparing Testing and Dynamic Testing Techniques for the Crosswind Sensitivity of Road Vehicles; SAE SP-1145. pp. 39-45.

[7] Bearman P.W. and Mullarkey S.P. (1994). - Aerodynamic Forces on Road Vehicles due to Steady Side Wind and Gust, Conference on Vehicle Aerodynamics, Loughborough, pp. 4.1-4.12.

[8] Davis J.P. (1982). - Wind Tunnel Investigation of Road Vehicle Wakes, $\mathrm{PhD}$ thesis, University of London.

[9] GARRY K.P. and COOPER K.R. (1986). - Comparison of Quasi-Static and Dynamic Wind Tunnel Measurements on Simplified Tractor-Trailer, Journal of Wind Engineering and Industrial Aerodynamics, $\mathrm{N}^{\circ} 22$, pp. 185-194.

[10] Chometon F. and Gillieron P. (1997). - Analysis of Unsteady Wakes by Image Processing in Automotive Aerodynamics, Fifth Triennial International Symposium on Fluid Control, Measurement and Visualization, September 1-4, Hayama, Japan.

[11] DOMINY R.G. and RYAN A. (1999), - An Improved Wind Tunnel Configuration for the Investigation of Aerodynamic Cross Wind Gust Response, SAE paper 1999-01-0808. International Congress, Detroit, USA.

[12] Minato K., Ryu H. and Kobayashi T. - Aerodynamics of Road Vehicles in Tunnels - Flow Visualization Using the Laser Light Sheet Method and its Digital Image Processing, Vehicle Aerodynamics, SAE International, PT-49, pp. 199-207, ISBN 1-56091-594-3.

[13] Marcu B. and Browand F. (1999). - Aerodynamic Forces Experienced by a 3-Vehicle Platoon in a Crosswind, SAE paper 1999-01-1324, International Congress, Detroit, USA.

[14] Taeyoung H., Sumantran V., Harris C., Kuzmanov T., Huebler M. and ZAK T. (1996). - Flow-Field Simulations of Three Simplified Vehicle Shapes and Comparisons with Experimental Measurements, SAE paper 960678 , International Congress, Detroit, USA.

[15] Ahmed S.R., Ramm R. and Faltin G. (1984). - Some Salient Features of the Time-Average Ground Vehicle Wake, SAE Technical Paper Series 840300, Detroit.

[16] Gillieron P. and Chometon F. (1999). - Modelling of Stationary Three-Dimensional Separated Air Flows around an Ahmed Reference Body. Third International Workshop on Vortex, ESAIM, Proceedings, Vol. 7, 173-182, http ://www.emath.fr/proc/Vol.7/.

[17] Perzon S., Janson J. and Höglin L. (1999). - On Comparisons between CFD Methods and Wind Tunnel Tests on a Bluff Body, SAE paper 1999-01-0805, International Congress, Detroit, USA.

[18] Gillieron P. (1999). - Potentiel et limites de l'utilisation de la simulation numérique en aérodynamique automobile, 14ème Congrès Français de Mécanique, Toulouse, 30 août-3 septembre 1999, Toulouse.

[19] OKumura K. and Kuriyama T. - Practical Aerodynamic Simulations (Cd, Cl, Cym) Using a Turbulence Model and $3^{\text {rd }}$-Order Upwind Scheme, SAE paper 950629, Vehicle Aerodynamics, SAE International, PT-49, pp. 385-397. ISBN 1-56091-594-3. 